\title{
Élisabeth SCHNEIDER
}

\section{Y A-T-IL UNE LITTERACIE ADOLESCENTE?}

Résumé : Le questionnement sur la littéracie est renouvelé par les nouveaux dispositifs d'écriture que sont par exemple le mobile et les réseaux sociaux numériques. Pour penser la complexité de la place de l'écriture dans la vie adolescente, il faut s'appuyer sur les apports de la géographie et des SIC pour penser respectivement la spatialité des situations d'écriture et les processus de production et de circulation des écrits. L'individuation et la socialisation adolescente mises en œuvre avec l'écriture peuvent se penser par ailleurs de manière fructueuse avec la médiance. La richesse des usages de l'écriture par les adolescents ouvre la voie à une géographie de l'écriture et une perspective littéracienne renouvelée.

Mots-clés : Littéracie adolescente- écriture adolescente- médiance- ethnographie de l'écriture- réseau social numérique- spatialité de l'écriture- écriture numérique

\section{INTRODUCTION}

Le questionnement sur la littéracie est aujourd'hui renouvelé dans la mesure où l'écriture s'inscrit dans de nouveaux dispositifs sociotechniques et de nouveaux modes d'élaboration sociale et individuelle. Les adolescents étant pour leur part nés " dans une société avec internet ${ }^{2} »$, ils sont souvent considérés comme ayant une familiarité, des aptitudes voire des compétences plus grandes que leurs aînés avec le numérique, tout en étant encore dans une culture de l'écrit, que ce soit dans la sphère privée ou scolaire. Leurs activités, leur rapport au monde, leurs pratiques culturelles, communicationnelles mais aussi leurs pratiques d'information sont à des degrés divers de manière indéniable liés aux outils numériques. Leurs écrits sont souvent qualifiés négativement: les écrits sur papier seraient devenus de plus en plus laborieux, parce qu'étrangers à la culture adolescente, en particulier ceux demandés à l'école. Les SMS seraient une forme appauvrie d'une communication relevant d'un " oral écrit », les contenus seraient réduits à la fonction phatique. Cependant, leurs pratiques d'écriture élargies à d'autres outils que le papier, à des espaces numériques organisant de nouveaux agencements matériels, symboliques et techniques tout en conservant par ailleurs des formes traditionnelles, engagent à soulever la question de la compréhension de ces écrits adolescents contemporains. Ainsi peut-on et comment cerner les contours d'une littéracie adolescente?

J'interrogerai le concept de littéracie, à partir de la prise en compte de la spatialité ${ }^{3}$ des pratiques d'écriture et de la notion de

${ }^{1}$ La nouveauté n'est pas seulement technique- écrire avec un smartphone par exemple- elle réside, on le verra, dans les agencements particuliers entre objets techniques, activités et acteurs.

${ }^{2}$ Avec tout ce que cette expression peut comporter d'approximations.

${ }^{3}$ La spatialité s'entend comme une des dimensions de la réalité et prenant en compte les relations que l'on établit avec cette dernière, qu'il s'agisse de la page de 
situation pour ensuite mettre en évidence à partir d'exemples issus d'une enquête, certains points saillants de l'écriture adolescente : l'écriture de SMS et celle sur Facebook. Je proposerai ensuite de considérer de manière plus globale les processus à l'œuvre dans l'interaction entre l'adolescent et les dispositifs sociotechniques par l'écriture en m'appuyant sur le concept de médiance.

\section{Enquêter sur les pratiques d'écriture}

Cette recherche a eu lieu dans le cadre d'une thèse dont l'objectif était de comprendre la place de l'écriture dans la vie adolescente aujourd'hui, entre papier et numérique, quelles que soient les activités, les moments, les lieux, les finalités (Schneider, 2012a). L'enquête ethnographique ${ }^{5}$ commencée à l'automne 2010 et terminée en juin 2012 a consisté tout d'abord à rencontrer des adolescents scolarisés en classe de seconde $^{6}$ sur le principe de l'interconnaissance. Au fur et à mesure des accords donnés, ils ont été suivis sur Facebook, sur un forum, en classe, à l'internat, en transport en commun et à domicile? . J'ai pris les lignes de bus scolaires régionaux matin et soir, ai observé les adolescents aux arrêts, pendant les trajets, discuté avec eux de manière informelle à la sortie des lycées, demandé à les rencontrer pour ensuite les suivre en cours. Ils m'ont pour la plupart « acceptée comme amie » sur Facebook et j'ai pu ainsi observer leur écriture avec ce dispositif de réseau social numérique $^{8}$. Les adolescents ont accepté plutôt facilement de me donner accès à leurs espaces et leurs écrits. Ils m'ont montré leurs SMS, expliqué dans quelles situations ils les faisaient, quelle valeur ces formes de communication écrites avaient pour eux et comment ils s'y prenaient pour ne pas être vus des enseignants. Mon parti était bien de prendre au sérieux ce qu'ils en disaient et de repérer de possibles logiques d'action, outillées par l'écriture.

l'écran, des lieux dans lesquels on situe son action (chambre, lycée, rue, etc.). L'espace n'est pas d'un point de vue géographique synonyme de « cadre délimitant l'action ».

${ }^{4} \mathrm{Je}$ propose de le faire à partir d'une enquête ethnographique menée pendant quinze mois auprès d'adolescents dans une perspective croisée entre géographie sociale et sciences de l'information et de la communication en particulier dans deux domaines émergents : en mobilité, et/ou sur des réseaux numériques dits sociaux

${ }_{6}^{5} \mathrm{Je}$ reviendrai sur cette qualification.

${ }^{6}$ L'entrée au lycée est à plusieurs titres l'occasion de nouvelles pratiques et d'un développement spécifique d'usages en raison du changement d'organisation de la vie adolescente.

${ }^{7}$ A la fin de l'enquête, les premiers rencontrés entrent en Terminale, les derniers en première.

${ }^{8}$ Les outils utilisés ont été un carnet de terrain, un enregistreur, un mobile pour prendre des photos et des captures d'écran permettant une collecte de données hétérogène et dense faite d'observations de terrains, de traces de conversations, de photos, d'écrits, etc. 


\section{LE CONCEPT DE LITTERACIE : UN OUTIL EPISTEMOLOGIQUE?}

Redonner à la littéracie toute sa complexité

Quand Goody définit la littéracie, il s'agit bien d'autre chose que des compétences liées à l'écriture et à la lecture. Même si cette perspective qui est celle de 1'Unesco est aujourd'hui essentielle sur les questions d'apprentissage, elle n'est pas celle qui permet de comprendre comment l'individu médie son rapport au monde par l'écriture, de manière complexe. Je définirai ainsi, à la suite de Goody, mais aussi des travaux en anthropologie de l'écriture (Fraenckel, Modj, 2010), la littéracie comme l'ensemble des pratiques, des gestes, des objets mais aussi des valeurs qui sont mobilisés par l'usage de l'écriture et la manière dont ceux-ci s'inscrivent dans des activités et sont socialisés (Privat, $2007^{9}$ ). L'ordinateur en est alors un des derniers avatars (Goody, 2007). Nous pouvons y ajouter le téléphone portable et les outils tels que les tablettes. L'écriture est une technique transversale à de multiples dispositifs. Poser la question du renouvellement de la réflexion sur la littéracie ne se fait pas uniquement à propos du numérique mais plutôt à propos de la dynamique qui peut s'instaurer entre une écriture liée au numérique et des formes d'écrits persistantes sur papier, avec peut-être l'émergence de formes produites au croisement de l'un et l'autre dans une perspective de translittéracie (Schneider, 2012b).

La littéracie, peut avoir une valeur heuristique pour comprendre ce qui est en jeu dans les usages de l'écriture aujourd'hui si elle retrouve son potentiel d'identification et de qualification des processus d'interaction avec le monde par l'écriture. Comme tous les concepts qui ont eu une grande diffusion, elle est aujourd'hui bien souvent naturalisée. La littéracie dans la définition que nous avons donnée plus haut doit pouvoir donner lieu à une approche de la complexité des activités, objets, lieux, etc., de l'écriture. Comment aujourd'hui peut-on en rendre compte en particulier pour les adolescents?

\section{La littéracie et la spatialité}

Une des pistes possibles pour restituer à la littéracie son potentiel épistémique est de considérer les pratiques d'écriture comme les matérialisations provisoires de processus plus complexes qu'il faudrait chercher à faire émerger. Kaufmann souligne cet élément dans son analyse des pratiques de socialisation: une pratique, un comportement est un donné qui masque le processus qui l'a produit (Kaufmann, 2007). Pour étudier la diversité et la complexité du rôle de l'écriture dans la vie adolescente, ne faut-il pas aller à la recherche de ces éléments invisibles et tenter de remonter aux processus et à la complexité de l'agencement matériel et idéel ${ }^{10}$ qui les a rendues possibles ? L'écriture, dans sa double dimension de processus et de résultat de celui-ci, trame le réel, en le sémiotisant en particulier mais dans des configurations provisoires. Cet écueil de l'attention porté seulement à la concrétisation

${ }^{9}$ Préface de l'ouvrage de Jack Goody, Pouvoirs et savoirs de l'écrit, dans lequel l'anthropologue revient sur un certain nombre de critiques qui lui ont été faites.

${ }^{10} \mathrm{Cet}$ adjectif est utilisé en géographie pour désigne ce qui relève de la valeur, des représentations que l'on donne aux choses par opposition à matériel. 
des phénomènes et processus a été pointé en sociologie des usages à propos de l'étude des traces d'usages des techniques en général et du numérique en particulier (Denouël et Granjeon, 2011). Mais cela concerne aussi l'étude des écrits contemporains.

Par l'anthropologie de l'écriture, on sait qu'elle est une spatialisation du langage mais a $\mathrm{t}$ - on réellement exploré ce que la spatialité, préoccupation géographique, peut apporter à sa compréhension? Les sciences humaines et sociales ont, depuis la fin des années quatre-vingt, pris en compte ce que l'on nomme la spatialisation des sociétés. En effet, comme le temps, la technique et les symboles, l'es-pace est un élément qui participe à l'inscription humaine dans le monde, qui rend possibles les interactions ${ }^{11}$. Si l'on reprend cette question en la liant à l'écriture, on voit qu'elle est en fait présente dans la notion même de littéracie. Pour le dire simplement, écrire, c'est écrire quelque part. C'est situer son action, c'est gérer des distances, chercher à les réduire ou au contraire s'éloigner symboliquement. C'est une activité qui s'inscrit dans une situation (Lussault, 2000) qui concrétise un certain agencement matériel et symbolique : des objets, un moment, un ou des acteurs, des finalités, des techniques qui s'organisent en une configuration provisoire. Les adolescents vivent ainsi des situations d'écriture multiples dans une journée qui leur permettent d'entrer en relation avec le réel, avec les autres, et d'organiser leur expérience. Identifier et observer ces situations permettent de se donner les moyens de penser la richesse de la littéracie actuelle dans la variété de ses actualisations $^{13}$. La dimension spatiale permet, par ailleurs, de poser la question de ce qui est en jeu dans ce processus de médiation entre soi et la réalité par l'écriture. Conjointement, cela signifie qu'il faut aussi affronter la singularité des usages qui ne peut manquer d'émerger de la description précise des situations et des processus.

\section{L'ECRITURE ADOLESCENTE : QUELLES SITUATIONS ?}

$\mathrm{Au}$ fil de l'enquête, j'ai pu repérer des formes d'écrits bien connues- prises de notes en cours, copie de chansons, petits mots aux amis - et d'autres a priori peu ou pas encore étudiés - BD élaborée collectivement en cours à l'insu des enseignants par exemple, statuts sur Facebook. À partir d'un ensemble hétérogène et foisonnant qui émergeait des observations, la démarche ethnographique et sa description armée par l'écriture de recherche permettent de rendre compte et de construire des moyens de compréhension de la diversité des usages pour une même pratique $^{14}$. A partir de l'exemple des SMS et de Facebook, nous pourrons

11 Il faut partager un même espace pour communiquer et échanger.

12 Je reprends la notion de situation de la géographie sociale (Lussault, 2000) à la suite de Foucault.

${ }^{13}$ Béatrice Fraenckel, dans l'ouvrage Les lieux du savoir, dirigé par Christian Jacob, invite à s'intéresser aux tables à écrire des chercheurs, c'est une piste que j'ai poursuivie à propos des adolescents : décrivant les tables de travail des lycéens au lycée et à leur domicile (Fraenckel, 2011).

${ }^{14}$ En effet, l'usage est la pratique enrichie du sens que lui donne l'acteur. 
approcher cette densité et cette variété des usages de l'écriture par les adolescents.

\section{Écrire des SMS}

\section{Une pratique d'écriture, des usages.}

Avec leur téléphone connecté ou non à Internet, mais très majoritairement aujourd'hui avec un forfait SMS illimités, les adolescents écrivent beaucoup de SMS, pendant leurs déplacements qu'ils soient à pied, en bus, en tramway, en voiture avec leurs parents, seuls, avec des amis. Les SMS, faut-il le rappeler, sont des écrits courts envoyés à l'aide d'un mobile et ils commencent à être étudiés en tant qu'écrit. Dans la littérature ou dans les médias, ils sont abordés comme des phénomènes linguistiques de néographies ou comme illustrant la dimension communicationnelle exacerbee des mœurs adolescentes, souvent décrite comme relevant, de la fonction phatique : "T'es où ? ", «J'arrive ». Comment repérer les usages réels de cet écrit? Si on ne considère que l'écrit en lui-même, on pourrait analyser ces SMS, écrits en marchant, de la même manière que ceux envoyés en cours ou de leur chambre. Cette écriture omniprésente chez les adolescents est premièrement inédite par son nomadisme. L'observation courante montre à quel point les déplacements sont l'occasion d'envoyer des SMS, d'en consulter, d'écrire donc, dans des lieux et des moments où l'écriture n'était quasiment pas possible auparavant. Ils doivent donc être pris au sérieux parce qu'ils constituent probablement des ressources nécessaires pour agir. La littéracie adolescente se joue aujourd'hui en partie dans cette articulation entre la question de la mobilité ${ }^{5}$ et celle des SMS et que l'on peut prendre en compte en circonscrivant des situations d'écriture en mobilité.

\section{Écrire en situation de mobilité : outil et ressource}

Les mobilités lycéennes s'organisent entre l'établissement, le centre ville, des zones commerciales, les arrêts de bus, de tramway, les domiciles. Les lycéens qui arrivent en seconde découvrent une liberté d'action qu'ils n'avaient souvent pas au collège. Ils ont souvent des trajets plus longs en transports en commun, qu'il s'agisse de tramway ou de bus régionaux. Ils vont au lycée, se retrouvent, se déplacent ensemble, se séparent, etc. Cette organisation se fait en très grande partie par SMS. Je m'appuierai sur quelques exemples issus de l'enquête pour interroger ces arts d'écrire en mobilité.

Thomas et Corentin sont deux adolescents qui ont fréquenté le même collège mais sont dans deux lycées différents. Ils préservent leur relation amicale en se retrouvant le lundi midi pour « manger en ville ». Le vendredi soir, Thomas qui finit plus tôt, quitte son lycée en tramway et rejoint Corentin devant le sien pour partager un moment avant de se séparer. Dans la matinée, ils échangent des SMS pour vérifier que l'autre est disponible, pour préciser l'heure, savoir qui d'autre sera au rendez-

${ }^{15}$ La mobilité n'est pas que le déplacement, elle est la prise en compte de la valeur accordée à celui-ci. Un certain nombre de travaux s'intéressent à la mobilité adulte, en particulier aux rapports sociaux qui s'organisent avec ces mobilités (Capron et alii, 2005) mais les mobilités adolescentes sont encore peu investiguées. 
vous. A partir du moment où Thomas quitte son lycée, il envoie des SMS réguliers pour dire où il en est. Corentin lui écrit aussitôt quand il estime qu'il y a un retard. On voit que l'écrit articulé au déplacement joue un rôle essentiel pour fabriquer un espace commun et organiser la continuité de celui-ci au-delà des modifications matérielles : monter, descendre du bus, marcher, s'asseoir. Cette pratique est tout à fait récurrente chez les adolescents, filles comme garçons.

Carla et Juliette, elles, fréquentent un autre lycée, elles sont internes, partagent la même chambre. Elles utilisent les SMS très fréquemment, en cours, aux inter-cours, dans leurs déplacements le lundi et le vendredi pour regagner le lycée ou leur domicile. Juliette me dit ne pas aimer savoir Carla seule lorsqu'elle va prendre son train le vendredi soir, un peu tard. Le trajet en tramway et le quartier de la gare, le soir, quand il fait nuit, sont vécus comme insécurisants. Juliette accompagne alors Carla par SMS. Le contenu est similaire à celui des SMS mentionnés plus haut, pourtant la signification sociale en est différente. Il s'agit, ici, d'une situation de co-présence: par l'usage de l'outil numérique, elles sont ensemble et fabriquent un lieu ${ }^{16}$ social qui les sécurise. Plus, Carla m'explique qu'elle conserve les SMS que Juliette lui a envoyés et qu'elle relit plus tard comme autant de remémorations d'émotions partagées. Les SMS constituent alors une ressource individuelle et sociale au-delà de la situation de mobilité et de production de l'écriture.

L'adolescent est un acteur social qui s'insère ainsi dans des activités et interagit avec son environnement en utilisant des techniques pour s'individuer et se socialiser. L'écriture est une des techniques qu'il utilise. Il semble bien à partir de ces quelques exemples que l'écriture revêt des formes et des fonctions inédites dans ces situations de mobilité. Les écrits numériques que sont les SMS, sont à la fois des écrits-outils pour organiser, pour se rassurer et des écrits-traces pour servir de ressources ultérieures ou de supports pour d'autres activités.

\section{Écrire sur Facebook}

\section{Des écrits d'écran à des échelles diverses}

Au fil des deux ans de l'enquête, j'ai pu suivre une vingtaine de lycéens sur Facebook dont une dizaine beaucoup plus précisément. Ce réseau social numérique apparaît bien souvent comme un des pires outils du numérique que les adolescents ont investi. Les parents, les enseignants de mon enquête ont à propos de l'usage de Facebook les griefs suivants : les jeunes ont trop «d'amis » qui n'en sont pas, ils surexposent leur vie privée, ils publient sans réflexion, leurs commentaires et leurs statuts démontrent une pauvreté linguistique, relationnelle et réflexive, sans parler des pratiques transgressives telles que le cyberharcèlement. Pourtant, le temps qu'ils y passent, les liens Internet qu'ils partagent et, concernant mon objet, la quantité d'écrits qu'ils y déposent incitent à considérer avec sérieux ces usages. Que se joue-t-il précisément concernant la communication adolescente sur ce dispositif

${ }^{16}$ Le lieu est la plus petite unité spatiale qui met en relation des individus. Le numérique permet de fabriquer des lieux dans une métrique hertzienne et non plus topographique. 
sociotechnique ? Quel rôle spécifique joue l'écriture partout présente? Dans quelle mesure observe-t-on sur celui-ci des formes littéraciennes spécifiques?

Les pratiques des adolescents suivis ont évolué au fil de l'évolution du réseau social lui-même, de leur appropriation technique, et de leur construction identitaire. Ils s'y étaient inscrits pour la plupart en cours d'année de troisième et ont beaucoup écrit : ils ont- selon le vocabulaire utilisé sur le réseau- déposé des statuts, fait des commentaires, discuté, partagé des contenus, posté des liens, etc.

L'écriture sur Facebook ${ }^{17}$ résiste à̀ l'analyse. On peut considérer qu'on a affaire à des écrits d'écran ${ }^{18}$ contraints par un dispositif technique à la logique commerciale évidente et qui permet d'interagir sur un mode assez proche de l'oral avec des contenus souvent sans intérêt. Mais, dès qu'on y regarde de plus près, on se rend compte que dans l'espace d'une page web, on peut avoir des écrits papier numérisés et partagés puis commentés, des statuts adressés à un collectif plus restreint que le réseau des amis, un commentaire qui instaure une relation duelle, des propos tenus à partir du fil d'actualités, des commentaires qui, au contraire, portent sur des photos déposées plusieurs mois plus tôt. Les échelles d'interaction peuvent être diverses, on peut supposer alors que les usages de l'écriture varient autant qu'il y a de possibilités d'interactions.

Une adolescente de quinze ans que j'ai pu suivre pendant son année de seconde, dépose, de manière récurrente sur Facebook, des textes qui sont adressés à sa grand-mère décédée plusieurs mois auparavant. Dans l'extrait, le message est envoyé avec son mobile, est lu par son réseau d'amis, parmi lesquels certains « aiment» mais aucun ne commente (figure 1, infra).

Comment comprendre ces écrits donnés à lire sur une même interface et qui produisent différentes situations de communication?

Comment considérer celui qui écrit, sujet scripteur d'un nouveau genre ? L'analyse d'interactions sur quelques jours permet de repérer que, même si l'écran spatialise des relations scripturales comme si elles étaient unifiées, il s'agit bien pour les adolescents de situations distinctes

Figure 1 : Juliette,

Facebook, janvier 2011

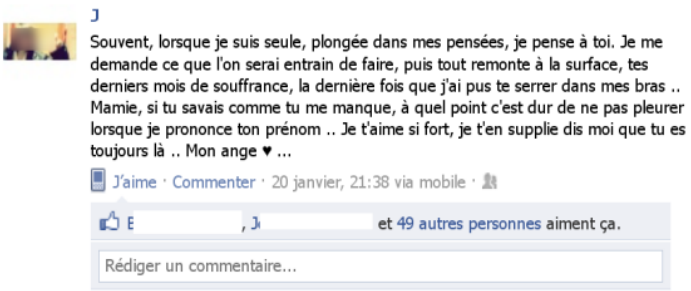

au moment de l'écriture. Les fonctions des écrits sont ainsi très diverses

${ }^{17}$ Ce réseau social numérique permet de manière générale aux individus de se créer un profil pour ensuite être en contact avec d'autres et communiquer, partager des contenus.

${ }^{18} \mathrm{Je}$ m'appuie sur la définition d'écrits d'écran élaborée par Souchier, Jeanneret, Le Marec qui prend en compte l'écrit comme outil et objet de manipulation par les médias informatisés (Souchier, Jeanneret, Le Marec, 2003) 
ainsi que le réseau réellement actualisé par les interactions. A partir de l'observation de celles-ci, il apparaît que le réseau d'amis n'est pas l'échelle pertinente pour étudier ce qu'ils font. Quand les adolescents écrivent, ils le font en pensant à quelques destinataires seulement. Sur les quelques trois cents amis, ils considèrent qu'ils ne communiquent réellement qu'avec une vingtaine. De la même manière, les échanges ont lieu de manière variable entre deux adolescents ou plus, faisant varier les échelles : ils actualisent des micro-réseaux sur des laps de temps variés. A ces échanges explicites s'ajoutent les adolescents qui lisent lęs écrits mais n'interviennent pas, dans une co-présence silencieuse ${ }^{19}$. Les situations de communication sont ainsi des configurations provisoires, multiples mais non éphémères, le réseau numérique les conservant par défaut.

\section{Quel rôle dans l'individuation et la socialisation adolescente ?}

Ces écrits déposés sur Facebook servent de support de socialisation, d'appropriation des comportements des groupes adolescents et à d'autres moments de ressources pour nourrir une relation. Ces dépôts, ces écrits stockés vont constituer des ressources ou des traces mémorielles, qui vont permettre de construire une stabilité du sujet adolescent contrairement à l'idée d'un flux permanent qui empêcherait la construction d'une stabilité quelconque (Stenger, 2011). Ces traces vont changer l'adolescent et comme il change, ces écrits changent de sens pour lui. Quand ils sont déposés, ils sont peut-être la trace d'un bon moment partagé mais au fil du temps, associés à d'autres, ils vont devenir par exemple la certification d'une relation qui s'est construite avec les autres adolescents. Ce travail de reprise des traces, supports, ressources est aussi traces et ressources pour les autres adolescents qui les voient et vont leur assigner une finalité encore différente. Au fil du temps, l'adolescent marque son environnement et en retour est affecté par lui : il dépose des écrits qui joueront un rôle pour les autres et en le faisant, il évolue lui-même. Ce processus est continu : les écrits qu'il a déposés auront un impact sur lui quand il les relira, quand d'autres les commenteront, modifiant alors à nouveau son regard.

On voit là toute la complexité du processus qui se met en route par l'usage de l'écriture. Celle-ci permet à l'adolescent d'entrer en relation avec son environnement en s'inscrivant dans la durée et en participant à sa construction identitaire. Ceci se fait de manière continue, sans que l'un ou l'autre ait la primauté et produisent ainsi un milieu relationnel. C'est ce qu'Augustin Berque nomme médiance (Berque, 2000). Je propose ainsi de considérer que l'adolescent, quand il écrit, produit un énoncé contraint par le dispositif utilisé : du cahier de cours à Facebook en passant par le mobile, qui devient alors une trace-empreinte d'un moment partagé, d'une émotion, mais aussi une matrice générant autre chose. Ces écrits sont capitalisés et peuvent constituer un matériau pour un travail de reprise ultérieur, social ou d'individuation. Ils sont autant de prises sur lequel l'adolescent pourra s'appuyer pour évoluer

${ }^{19}$ Je n'aborderai pas ici la question de l'usage du «like » emblématique de Facebook beaucoup plus varié dans ses significations qu'une approbation affective et qui mériterait d'être analysé aussi comme un écrit. 
mais le faisant, il aura contribué à agir sur le dispositif, sur la situation pour lui et pour les autres s'il s'agit d'un dispositif partagé.

\section{UNE LITTERACIE ADOLESCENTE A INVESTIGUER}

\section{Pratiques d'écriture transmédiatiques}

Dans les exemples précédents, même si j'ai cherché à insister sur la complexité des situations d'écriture, dont la description fine peut permettre d'appréhender la richesse d'usage de l'écriture, on pourrait objecter que les supports ont été considérés de manière juxtaposée. Cela l'a été pour faciliter la mise en évidence de formes littéraciennes nouvelles et particulièrement diffusées chez les adolescents. Cependant, dans le cadre de ma recherche, la pensée par cas a permis de restituer la richesse et la singularité des usages et de donner à voir comment l'écriture trame la vie d'un adolescent (Passeron, Revel, 2005). Ces adolescents écrivent aussi bien sur papier que sur support numérique, font circuler les écrits de l'un à l'autre (figure 2, BD élaborée en cours, partagée et commentée sur Facebook), les transforment, les stockent, les organisent, les indexent même, et ce de manière différenciée. Ces pratiques que l'on peut nommer transmédiatiques, à la suite des travaux de Jenkins (Jenkins, 2006) manifestent le developpement d'un capital littéracien (Privat, 2006). En effet, ils jouent des supports, des formes, des genres, des outils liés à l'écriture pour organiser leur expérience sociale et individuelle. En ce sens, je parlerai d'économie scripturale parce que les adolescents dans cette organisation font des choix. Les écrits produits sont le résultat d'arbitrages, de négociation, de gestion des contraintes. Cela ne signifie pas qu'ils le font toujours avec efficience mais ils dénloient des arts de faire complexes.

(insérer ici la fig.2)

Jalons pour une géographie de l'écriture?

Pour rendre opératoire le concept de littéracie, Jean-Marie Privat propose la notion de " coefficient de littéracie » (Privat, 2006). Il s'agit d'identifier dans les formes de l'écrit le degré de littéracie, à savoir lesquelles sont spécifiques d'un ordre de l'écrit. Si l'on observe les pratiques et les usages adolescents mentionnés ici, il semble bien que les écrits sur les réseaux sociaux qui mettent en œuvre des processus de socialisation et d'individualisation, écrits que j'ai nommés écrits-traces, écrits-empreintes ne sauraient avoir lieu sans l'écriture. Nous avons là des élaborations symboliques inédites, fondées sur l'interaction, dans des agencements permis et contraints par le dispositif technique, accessible à l'analyse à une échelle d'observation dont la pertinence est à définir. La spatialité est une caractéristique essentielle de ces écrits. De la même façon, la manière dont ces adolescents écrivent des SMS, les stockent, les relisent dans une visée de remémoration constituent des activités à fort coefficient de littéracie. On est là, il me semble, bien loin d'une caractérisation fréquente des SMS ou des écrits sur les RSN comme d'un " oral écrit », qui serait la preuve, entre autres, d'une obsession de la connexion typique des adolescents.

Pour poursuivre ce champ ouvert de la littéracie adolescente, on pourrait dessiner les contours d'une géographie de l'écriture, dans la mesure où la dimension spatiale de ces écrits ne saurait être négligée et où l'écriture sert aux adolescents à fabriquer des espaces, des lieux et à 
les délimiter mais aussi parce que ces écrits contemporains sont encore finalement très peu investigués dans leurs spécificités dont j'ai tenté de souligner quelques aspects. Je préciserai deux pistes dont les enjeux éducatifs et didactiques sont importants pour conclure ici : il s'agirait d'étudier par exemple comment s'élaborent des réseaux d'écrits. En effet, les écrits circulent d'un support à l'autre, sont documentarisés, travaillés, enrichis, s'organisent en constellations dans une perspective transmédiatique, et mettent en œuvre des habiletés translittéraciques (Thomas, 2007). Une autre piste serait l'étude des situations d'écriture, de leurs agencements matériels, symboliques, la manière dont sont mêlés numérique et support papier et la place qu'y occupent les adolescents, y compris physiquement.

\title{
CONCLUSION
}

Alors que le constat est parfois fait que le numérique consacre le retour de l'oralité, l'enquête ethnographique menée montre qu'au contraire pour les adolescents, l'écriture est à la fois un outil essentiel et une ressource pour des finalités complexes et variées. Leur vie est tramée de numérique, mais elle l'est tout autant d'écriture. De la même façon, cela remet en question la qualification de ces pratiques d'immatérielles ou de virtuelles: elles sont incarnées et les adolescents en sont pleinement sujets. La majorité des pratiques identifiées est invisible aux adultes, enseignants et parents. Ce que j'ai nommé l'économie scripturale l'est encore davantage puisqu'elle demande une perspective holiste des situations d'écriture, rendue possible ici par l'exigence épistémologique de l'ethnographie. Le concept de littéracie peut ainsi avoir une valeur heuristique dans la mesure où il retrouve son ampleur anthropologique.

\author{
Élisabeth SCHNEIDER \\ ESPE-UCBN \\ ESO Caen - UMR 6590
}

\begin{abstract}
The subject of literacy is renewed because of the new media and the new socio-technical systems, as mobile phones and social networks. To think the complexity of the role of writing in youth's life, we have to rely on the concept of « spatiality », of « situation », and to bring out the process of production and circulation of written. We'll consider the youth's individuation and socialisation implemented with writing with the concept of «mediance». The diversity of the youth's writing opens the path to a geography of writing and a renewed literacy.

Key-words : youth's writing- mediance- spatiality of writing- geography of writingdigital writing- social network- ethnography of writing
\end{abstract}

\section{Bibliographie}

Berque A. (2000, 1ère éd. 1990) Médiance, de milieux en paysages. Paris : Belin.

Capron G., Cortès G. \& Guétat-Bernard H. (2005) Liens et lieux de la mobilité. Ces autres territoires. Paris : Belin.

Coutant A. \& Stenger T. (dir.) (2011) Ces réseaux numériques dits sociaux - Hermès 59. 
Denouël J. \& Granjon F. (2011) (dir.) Communiquer à l'ère numérique. Regards croisés sur la sociologie des usages. Paris: Ed. Transvalor/Presses des Mines.

Goody J. (2007) Pouvoirs et savoirs de l'écrit. Paris : La Dispute.

Fraenckel B. \& Mbodj A. (2010) «Introduction. Les New Literacy studies, jalons historiques et perspectives actuelles »Langage et Société 133 (7-24).

Fraenckel B. (2011) «L'insaisissable table à écrire » - in : C. Jacob (dir.) Les mains de l'intellect. Les lieux du savoir 2 (117-124). Paris : Albin Michel.

Jenkins H. (2006) Convergence Culture. NY : New York University Press.

Kaufmann J. C. (2007) Ego, pour une sociologie de l'individu. Paris, Hachette.

Lussault M. (2000) «Actions" - in : J. Lévy et M. Lussault (dir.) Logiques de l'espace, esprit des lieux. Géographies à Cerisy (11-36). Paris : Belin.

Passeron J.-C. \& Revel J. (dir.), (2005) Penser par cas. Paris : Éditions de l'EHESS.

Privat J. M. (2006) «Un habitus littératien ? »-Pratiques 131-132 (125130).

Schneider E. (2012a) «L'écriture des adolescents, une démarche ethnographique pour rendre compte de la spatialisation des pratiques »-La géographie des enfants et des jeunes, Carnets de Géographes, 3.

http://www.carnetsdegeographes.org/carnets_terrain/terrain_03_05_Schneider.php

Schneider E. (2012b) «Pratiques d'écriture des adolescents et translittératie »- in: A. Serres (dir.) La translittératie en débat: regards croisés des cultures de l'information et des disciplines, séminaire GRCDI, 7 septembre 2012, URFIST de Rennes.

http://culturedel.info/grcdi/wp-content/uploads/2012/09/Seminaire-GRCDI-2012 texte-

E.Schneider.pdf

Schneider E. (2013) «Paysage scriptural adolescent, du papier au numérique » - in : H. Chalak, S. Fontaine, M. MassotLeprince et M. Fabre (2013) Education et formation, Actes du colloque doctoral international de l'éducation et de la formation, Nantes, 25-26 novembre 2011, Recherches en éducation, Hors-série $\mathrm{n}^{\circ} 5$.

http://www.recherches-en-education.net/spip.php?article148

Souchier E., Jeanneret Y. \& Le Marec J. (dir.) (2003) Lire, écrire, récrire - objets, signes et pratiques des médias informatisés. Paris : Bibliothèque Publique d'Information.

Thomas S. et al. (2007) "Transliteracy: Crossing divides» - First Monday, XII, 12, 3 décembre 2007.

http://firstmonday.org/ojs/index.php/fm/article/view/2060 